\title{
Upregulation of PAWR by small activating RNAs induces cell apoptosis in human prostate cancer cells
}

\author{
KAI YANG $^{1 *}$, JIE SHEN $^{2 *}$, SHAN-WEN CHEN $^{1}$, JIE QIN $^{1}$, XIANG-YI ZHENG $^{1}$ and LI-PING XIE ${ }^{1}$ \\ ${ }^{1}$ Department of Urology, The First Affiliated Hospital, Zhejiang University, Hangzhou, Zhejiang 310003; \\ ${ }^{2}$ Department of Pharmacy, Traditional Chinese Medical Hospital of Zhejiang Province, Hangzhou, Zhejiang 310006, P.R. China
}

Received November 1, 2015; Accepted January 7, 2016

DOI: 10.3892/or.2016.4582

\begin{abstract}
RNA activation (RNAa) is a promising discovery whereby expression of a particular gene can be induced by targeting its promoter using small double-stranded RNAs (dsRNAs) also termed small activating RNAs (saRNAs). We previously reported that several small dsRNAs targeting the PRKC apoptosis WT1 regulator (PAWR) promoter can upregulate PAWR gene expression effectively in human cancer cells. The present study was conducted to evaluate the antitumor potential of PAWR gene induction by these saRNAs in prostate cancer cells. Promisingly, we found that upregulation of PAWR by saRNA inhibited the growth of prostate cancer cells by inducing cell apoptosis which was related to inactivation of the $\mathrm{NF}-\kappa \mathrm{B}$ and Akt pathways. The decreased anti-apoptotic protein $\mathrm{Bcl}-2$ and activation of the caspase cascade and poly(ADP-ribose) polymerase (PARP) also supported the efficacy of the treatment. Overall, these data suggest that activation of PAWR by saRNA may have a therapeutic benefit for prostate and other types of cancer.
\end{abstract}

\section{Introduction}

Prostate cancer is the most common cancer in males in economically developed countries. In 2014, there were an estimated 233,000 new cases of prostate cancer and 29,480 related deaths in the US (1). Metastatic castration-resistant prostate cancer is the primary cause of death for most patients. Unlike

Correspondence to: Dr Shan-Wen Chen, Department of Urology, The First Affiliated Hospital, Zhejiang University, 79 Qingchun Road, Hangzhou, Zhejiang 310003, P.R. China

E-mail: supergene@163.com

*Contributed equally

Abbreviations: RNAa, RNA activation; saRNA, small activating RNA; RNAi, RNA interference; dsRNA, double-stranded RNA; PAWR, PRKC apoptosis WT1 regulator; TSG, tumo-suppressor gene; MTT, 3-(4,5-dimethylthiazol-2-yl)-2,5-diphenyltetrazolium bromide; $\mathrm{nM}, \mathrm{nmol} / \mathrm{l}$

Key words: RNA activation, small activating RNA, PAWR, prostate cancer, cell apoptosis the majority of solid cancers, prostate cancer usually shows a poor response to chemotherapy. Therefore, more effective strategies for the treatment of castration-resistant prostate cancer are urgently required.

Therapeutics based on RNA interference (RNAi) have become powerful and ideal methods for the treatment of many diseases including cancer which are mainly caused by overactive oncogenes due to the high specificity, high efficacy and low toxicity of the RNAi trigger - small doublestranded RNAs (dsRNAs) (2-4). However, there are many types of cancer that are mainly caused by complete inactivation or reduced expression of tumor-suppressor genes (TSGs). Notably, new evidence has emerged that synthetic small dsRNAs induce sequence-specific transcriptional gene activation of E-cadherin, p2 $1^{\mathrm{WAF} 1 / \mathrm{CIP} 1}$ and VEGF by targeting specific regions in their gene promoters (5). This phenomenon has been termed RNA-induced gene activation (RNAa) and such dsRNAs as small activating RNAs (saRNAs) (5). Their observation was supported by subsequent studies which suggest that RNAa may be a general and conserved phenomenon of gene regulation (6-14). Moreover, several studies have demonstrated that restoration of p21 expression by saRNAs in different cancer cells could inhibit cell proliferation and tumor growth (15-20). Thus, RNAa holds great promise as an alternative to traditional vector-based systems to activate target genes and would supplement RNA-mediated gene silencing to broaden the gene pool susceptible to regulation by small RNAs.

The human PAWR (PRKC apoptosis WT1 regulator) gene, whose other aliases include PAR4 and Par-4, is located on chromosome 12q21 and encodes a leucine zipper domain protein first identified in prostate cancer cells undergoing apoptosis induced by an exogenous insult $(21,22)$. Mutations in the PAWR gene have not been reported in cancer cells, and endogenous PAWR expressed in normal and cancer cells does not, by itself, cause apoptosis. However, inhibition of endogenous PAWR with antisense oligodeoxynucleotides, a dominant-negative leucine zipper domain or RNAi, precludes apoptosis by exogenously applied agents, thus indicating that functional PAWR protein is essential for apoptosis via diverse cell death pathways (23-25). More importantly, ectopic PAWR overexpression is sufficient to induce apoptosis in most cancer cells in vitro and growth inhibition of prostate cancer xenografts in nude mice, but not in normal or immortalized 
cells $(23,26)$. Therefore, PAWR is an ideal target and a candidate TSG for RNAa.

Our previous study demonstrated that several small dsRNAs targeting the PAWR promoter can upregulate PAWR gene expression effectively in human cancer cells (14). In the present study, we investigated the antitumor effects of dsPAWR-433 on prostate cancer cell lines and found that upregulation of PAWR by saRNA inhibited the growth of prostate cancer cells by inducing cell apoptosis.

\section{Materials and methods}

dsRNA design and synthesis. The sequence of dsPAWR-433 [S, 5'-AAU ACG GUC UUG UAC UUA A (dT)(dT)-3'; AS, 5'-UUA AGU ACA AGA CCG UAU U (dT)(dT)-3'] was designed as previously described (17); and the control dsRNA [dsCon: S, 5'-ACU ACU GAG UGA CAG UAG A (dT)(dT)-3'; AS, 5'-UCU ACU GUC ACU CAG UAG U (dT)(dT)-3'] is the same as the dsCon-2 which was specifically designed by Li et al to lack homology to all known human sequences (5). All dsRNAs were chemically synthesized by GenePharma (Shanghai, China) with dTdT-3' overhangs.

Cell culture and transfection. The human prostate cancer cell lines DU145 and PC3 were obtained from the Shanghai Institute of Cell Biology, Chinese Academy of Science. The cells were cultured in RPMI-1640 medium supplemented with $10 \%$ heat-inactivated fetal bovine serum, penicillin $(100 \mathrm{U} / \mathrm{ml})$ and streptomycin $(100 \mathrm{mg} / \mathrm{l})$ in a humidified atmosphere containing $5 \% \mathrm{CO}_{2}$ maintained at $37^{\circ} \mathrm{C}$. The day before transfection, the cells were plated in growth medium without antibiotics at a density of 30-40\%. Transfections of dsRNAs were carried out using Lipofectamine 2000 (Invitrogen, Carlsbad, CA, USA) according to the manufacturer's protocol and lasted for 24,48 or $72 \mathrm{~h}$. Cell images were captured using a phase-contrast microscope at a magnification of $\times 100$ (Olympus, Japan).

Cell growth/viability assay. Proliferation of cells was determined by the 3-(4,5-dimethylthiazol-2-yl)-2,5-diphenyltetrazolium bromide (MTT) assay (16). Approximately 5,000-10,000 cells were plated in each well of a 96-well plate. After overnight incubation, the cells were treated with the appropriate dsRNAs for 24,48 or $72 \mathrm{~h}$. At the various times after treatment, $20 \mu \mathrm{l}$ MTT $(5 \mathrm{mg} / \mathrm{ml})$ was added to each well and the plates were incubated at $37^{\circ} \mathrm{C}$ for $4 \mathrm{~h}$. After that, the crystals were dissolved in $150 \mu \mathrm{l}$ of dimethyl sulfoxide at room temperature. Absorbance was measured at $490 \mathrm{~nm}$ in an absorbance reader (MRX II; Dynex Technologies, Chantilly, VA, USA). The reduction in viability of each group was expressed as a percentage of the mock group, which was considered to be $100 \%$ viable.

Real-time quantitative RT-PCR. Total RNA was extracted from cells using TRIzol (Invitrogen) and reverse transcribed using oligo(dT) primers. The resulting cDNA was amplified in a real-time PCR system (ABI Prism 7500; Applied Biosystems, Foster City, CA, USA) using the DNA-binding dye SYBRGreen I (Invitrogen) for detection of PCR products. Values are expressed as fold-difference compared with the mock group.
Primer sequences for PAWR were: 5'-GCCGCAGAGTGCTT AGATGAG-3' (forward) and 5'-GCAGATAGGAACTGC CTGGATC-3' (reverse) and; for GAPDH were: 5'-AAGAA GGTGGTGAAGCAGGC-3' (forward) and 5'-TCCACCACCC TGTTGCTGTA-3' (reverse).

Western blot analysis. Protein extraction and western blot analysis were carried out according to a previously described method (16). The primary and secondary antibodies were all purchased from the Cell Signaling Technology (Beverly, MA, USA).

To determine $\mathrm{NF}-\kappa \mathrm{B}$ cellular localization, nuclear and cytoplasmic proteins were isolated from the cells using a cell fractionation kit (KeyGen, Wuhan, China). NF- $\kappa \mathrm{B}$ expression in the nuclear and cytoplasmic compartments was determined by immunoblot analysis as described above.

Detection of apoptotic cells by flow cytometry. A quantitative assessment of apoptosis was carried out by determining the percentage of cells with highly condensed or fragmented nuclei. Cells were plated in 6-well plates and incubated overnight before treatment. Then, cells were harvested at $72 \mathrm{~h}$ after dsRNA treatment, washed twice with pre-chilled phosphate-buffered saline (PBS), and resuspended in $100 \mu \mathrm{l}$ $1 \mathrm{X}$ binding buffer at a concentration of $1 \times 10^{6}$ cells $/ \mathrm{ml}$. Double staining with fluorescein isothiocyanate (FITC)-conjugated Annexin V and propidium iodide (PI) (Annexin V-FITC apoptosis detection kit; BD Biosciences, San Jose, CA, USA) was performed in accordance with the manufacturer's protocol. Cell apoptosis analysis was performed within $1 \mathrm{~h}$ using the Beckman Coulter FC500 Flow Cytometry system with CXP Software (Beckman Coulter, Fullerton, CA, USA).

Statistical analysis. All values are expressed as means \pm SD. Statistical significance was compared between treatment groups and controls using the Student's t-test. $\mathrm{P}<0.05$ was considered to indicate a statistically significant result.

\section{Results}

dsPAWR-433 induces PAWR gene activation in prostate cancer cells. We previously reported that several dsRNAs targeting the PAWR gene promoter at position $-433-435$ relative to the transcription start site (dsPAWR-433-435, Fig. 1A) had the ability to activate PAWR expression in T24 bladder cancer cells (14). In the present study, we investigated whether dsPAWR-433 could induce PAWR gene expression in prostate cancer cells. Fifty nmol/1 (nM) dsPAWR-433 and a non-specific control dsRNA (dsCon) were transfected into DU145 human prostate cancer cells and PAWR expression levels were evaluated 48 and $72 \mathrm{~h}$ later. Compared with the mock and dsCon groups, dsPAWR-433 caused a $>3$-fold induction in the PAWR mRNA level in the DU145 cells (Fig. 1B). Induction of PAWR was also confirmed by western blot analysis and the elevated levels of PAWR protein were strongly correlated to the increase in PAWR mRNA expression (Fig. 1C and D).

Transfection of dsPAWR-433 was also performed in another human prostate cancer cell line PC3. As shown in Fig. 2, dsPAWR-433 transfection resulted in a >2-fold induction of PAWR gene expression in the PC 3 cells. 
A
dsPAWR-433

5'-AAUACGGUCUUGUACUUAAdTdT-3' 3'-dTdTUUAAGUACAAGACCGUAUU-5

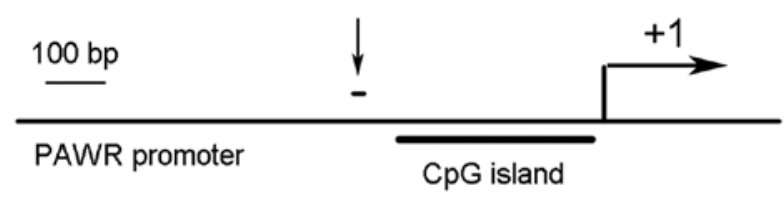

C

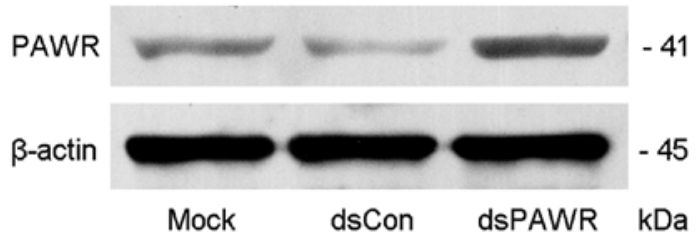

B
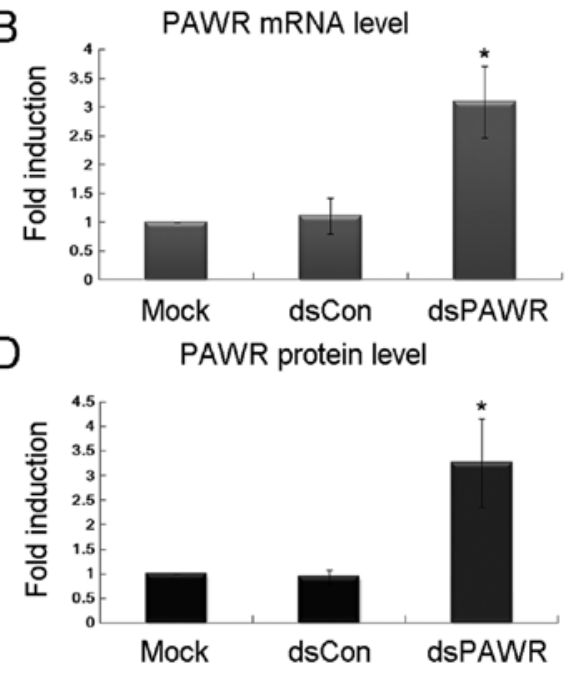

Figure 1. dsPAWR-433 upregulates PAWR gene expression in prostate cancer cell line DU145. (A) A schematic representation of the PAWR promoter with its CpG island, transcription start site, and dsRNA target. (B) DU145 cells were transfected with $50 \mathrm{nM}$ dsRNAs for $48 \mathrm{~h}$. mRNA expression levels of PAWR and GAPDH were detected by real-time RT-PCR, and the results were normalized to GAPDH and presented as the mean \pm SD of three independent experiments. (C) DU145 cells were transfected with $50 \mathrm{nM}$ dsRNA for $72 \mathrm{~h}$. PAWR and $\beta$-actin expression levels were detected by western blotting. A representative blot is shown from three independent experiments with identical results. (D) Relative protein level was determined by quantifying western blotting membrane band intensity. The PAWR protein expression levels were normalized to $\beta$-actin and the results are presented as the mean \pm SD of three independent experiments. ${ }^{*} \mathrm{P}<0.05$ compared with the mock.

A

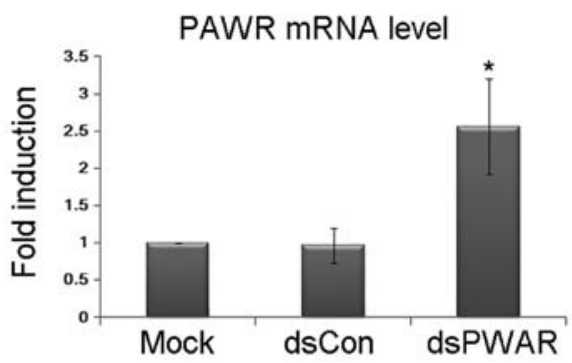

B

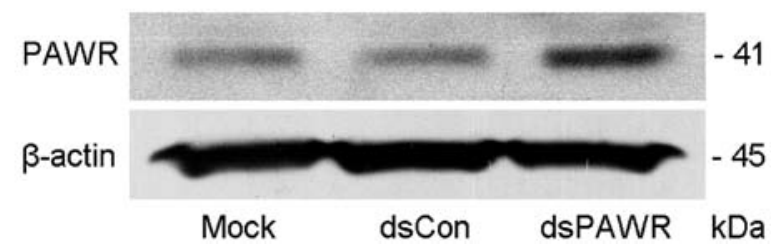

Figure 2. dsPAWR-433 upregulates PAWR gene expression in human prostate cancer cell line PC3. (A) PC3 cells were transfected with $50 \mathrm{nM}$ dsRNAs for $48 \mathrm{~h}$. mRNA expression levels of PAWR and GAPDH were detected by real-time RT-PCR, and the results were normalized to GAPDH and presented as the mean \pm SD of three independent experiments. ${ }^{*} \mathrm{P}<0.05$ compared with the mock. (B) PC3 cells were transfected with $50 \mathrm{nM}$ dsRNA for $72 \mathrm{~h}$. PAWR and $\beta$-actin expression levels were detected by western blotting. A representative blot is shown from three independent experiments with identical results.

dsPAWR-433 inhibits prostate cancer cell growth and viability. Ectopic PAWR overexpression has been shown to induce growth inhibition in most cancer cells in vitro (26). In the present study, we investigated whether the upregulation of PAWR by saRNA has similar effects on prostate cancer cells. DU145 prostate cancer cells were transfected with $50 \mathrm{nM}$ dsPAWR-433 and dsCon for 48 or $72 \mathrm{~h}$, and the dsPAWR433-transfected cells gradually displayed growth inhibition and cell shrinkage (Fig. 3). Moreover, evidently decreased cell density and more floating dead cells were observed in the dsPAWR-433-treated group (Fig. 3). These morphological changes were also observed in the prostate cancer cell line PC3 (Fig. 4).

Then, the effects of dsPAWR- 433 on the proliferation and viability of human prostate cancer DU145 cells were determined at varying concentrations and times (24-72 h) by MTT assay. As shown in Fig. 5A, the effects of dsPAWR-433 on cell viability, which were dose- and time-dependent, occurred within $48 \mathrm{~h}$ and at dsRNA concentrations as low as $5 \mathrm{nM}$. Compared with the mock and dsCon transfections, reduction in viability of the DU145 cells following dsRNA treatment at concentrations of $1-50 \mathrm{nM}$ after $48 \mathrm{~h}$ ranged from 3.6 to $29.5 \%$, whereas after $72 \mathrm{~h}$ this ranged from 13.0 to $72.6 \%$ (Fig. 5A). Accordingly, lower concentrations of dsPAWR-433 (5-25 nM) could also elevate the PAWR expression and its effects also appeared to be dose-dependent (Fig. 5B).

dsPAWR-433 induces cell apoptosis in prostate cancer cells. The antitumor ability by ectopic PAWR overexpression is related to its essential role in inducing apoptosis via diverse cell death pathways (23-25). Thus, we investigated the relationship between dsPAWR-433-mediated loss of cell viability and apoptosis by flow cytometric analysis of DU145 cells labeled with PI and Annexin V. We found that dsPAWR-433 caused evident apoptosis in the DU145 cells at $72 \mathrm{~h}$ following treatment. The number of early apoptotic cells (LR quadrant) increased to $40 \%$ and the number of late apoptotic cells (UR quadrant) increased to nearly 20\% (Fig. 6A and B). These data also showed that dsPAWR-433 treatment resulted in not only apoptosis but also tiny cell necrosis, which may be a secondary event in the apoptotic process.

Caspase- 3 and poly(ADP-ribose) polymerase (PARP) play central roles in apoptosis. Accordingly, we observed that the 

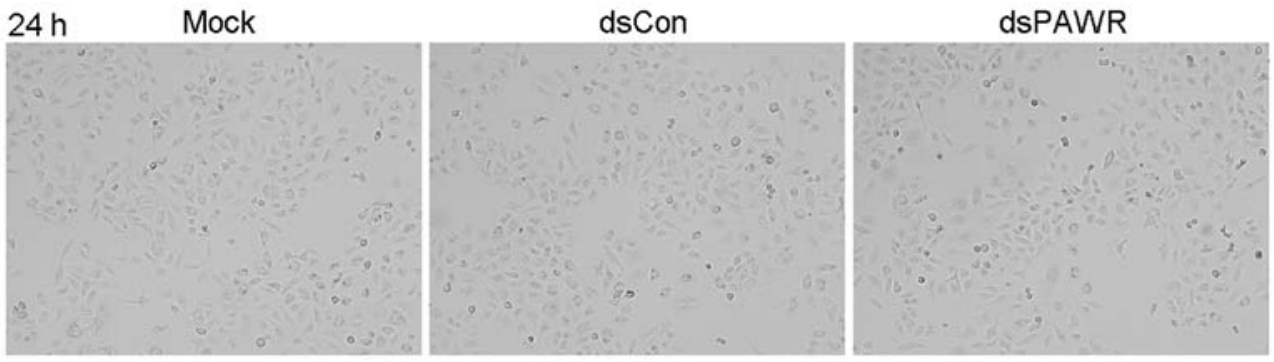

$48 \mathrm{~h}$
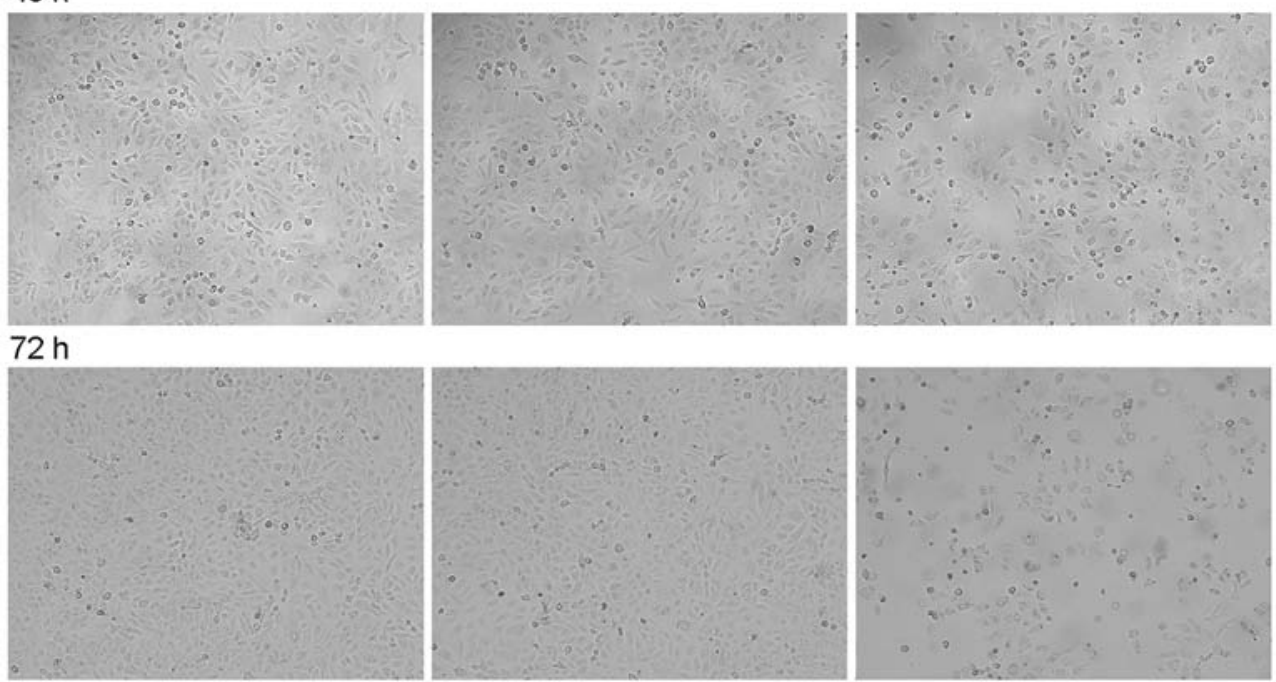

Figure 3. dsPAWR-433 induces growth inhibition of DU145 cells. Cells were transfected with $50 \mathrm{nM}$ dsRNAs or mock. Cell images were captured at 24,48 and $72 \mathrm{~h}$ after transfection at a magnification of x100. dsPAWR-433-transfected cells are less dense and have more dead cells than the controls.
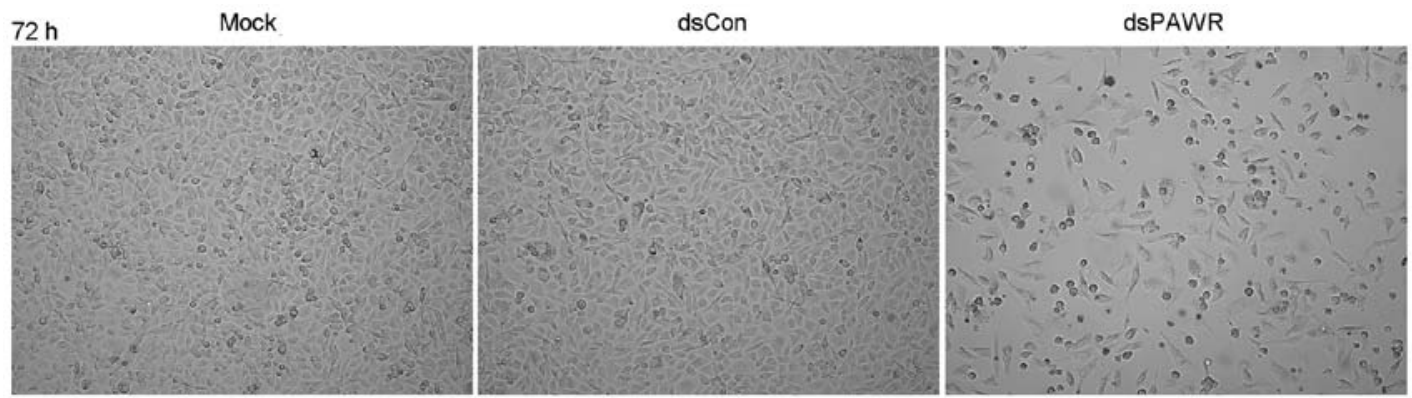

Figure 4. dsPAWR-433 induces growth inhibition of PC 3 cells. Cells were transfected with $50 \mathrm{nM}$ dsRNAs or mock. Cell images were captured at $72 \mathrm{~h}$ after transfection at a magnification of x100. dsPAWR-433-transfected cells are less dense and have more dead cells than the controls.
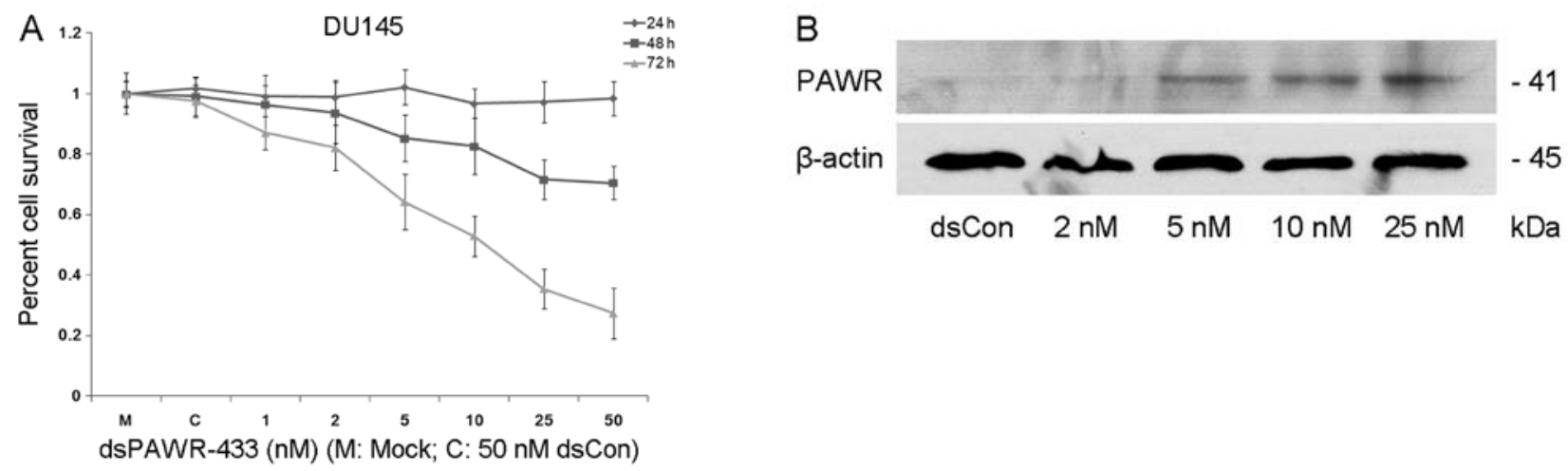

Figure 5. (A) dsPAWR-433 inhibits the viability of DU145 cells in a dose- and time-dependent manner, as assessed by the MTT assay. Reduced cell viability was noted after dsPAWR-433 treatment $(1-50 \mathrm{nmol} / \mathrm{l})$ at 24,48 and $72 \mathrm{~h}$. Data are presented as means $\pm \mathrm{SD}(\mathrm{n}=8)$. (B) DU145 cells were treated with dsPAWR433 at the indicated concentrations for $72 \mathrm{~h}$. PAWR and $\beta$-actin expression levels were detected by western blotting. A representative blot is shown from three independent experiments with identical results. 
A
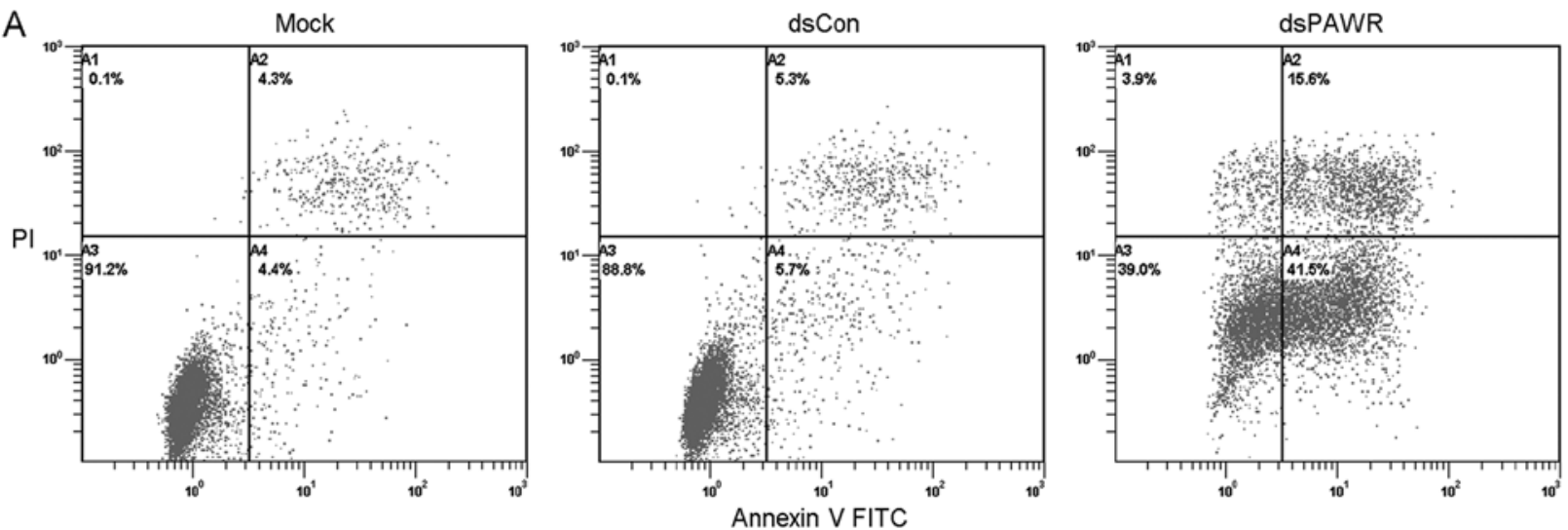

B

dsPAWR-433 induced cell apoptosis

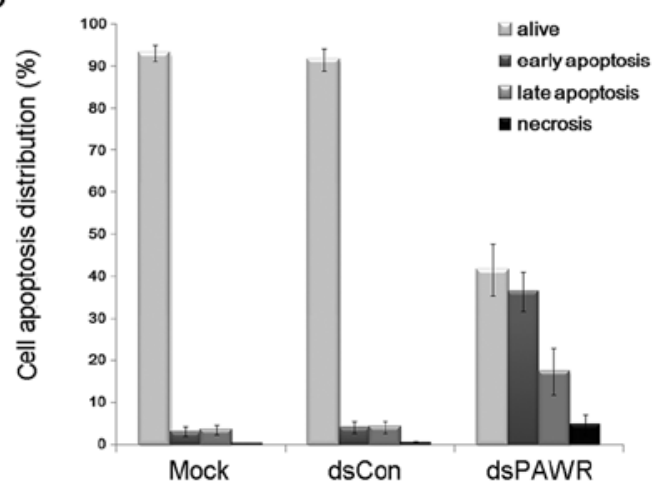

C

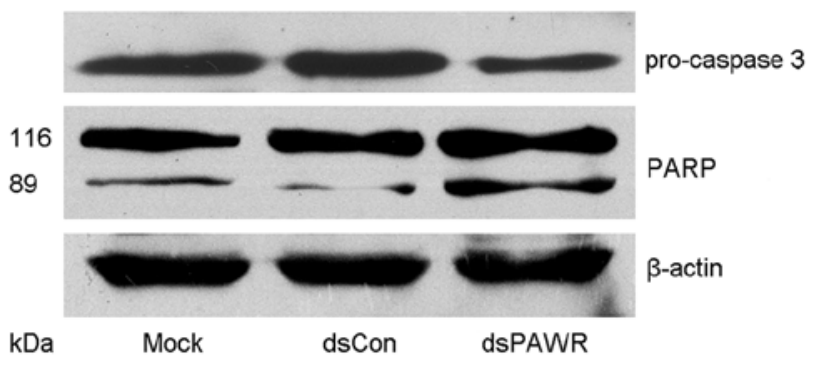

Figure 6. dsPAWR-433 induces cell apoptosis in DU145 cells. Cells were transfected with 50 nM dsRNA for 72 h. Representative images and blots from three independent experiments with identical results are shown. (A) dsPAWR-433 resulted in apoptosis in the DU145 cells as detected by flow cytometry using a double-staining method with fluorescein thiocyanate-conjugated Annexin V and propidium iodide. Annexin V-stained cells indicates the early apoptotic cells, whereas Annexin V + propidium iodide-stained cells are the late apoptotic cells. (B) Flow cytometric data were analyzed to compare cell apoptotic populations (means \pm SD from three independent experiments). Percentages of alive, apoptotic and necrotic cells are shown respectively. (C) dsPAWR-433 treatment activated caspase-3 and poly(ADP-ribose) polymerase (PARP) in the DU145 cells.

A

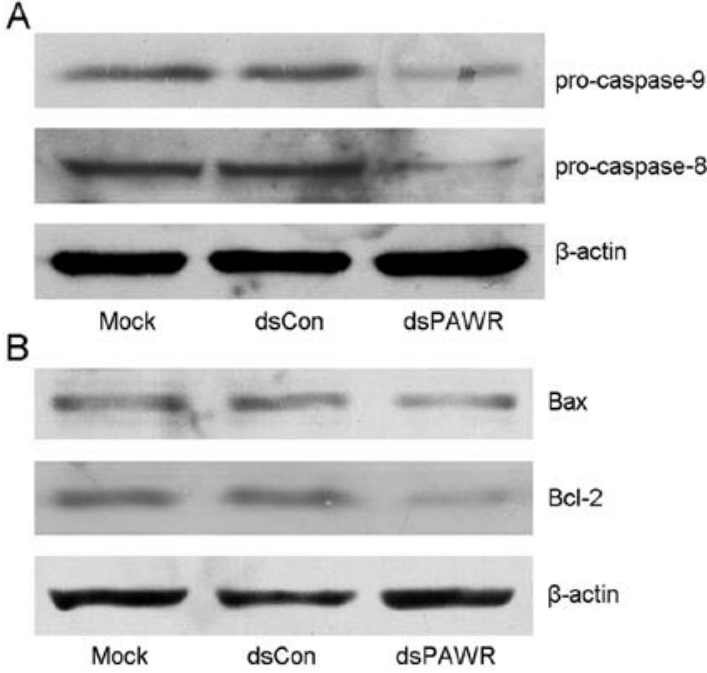

C

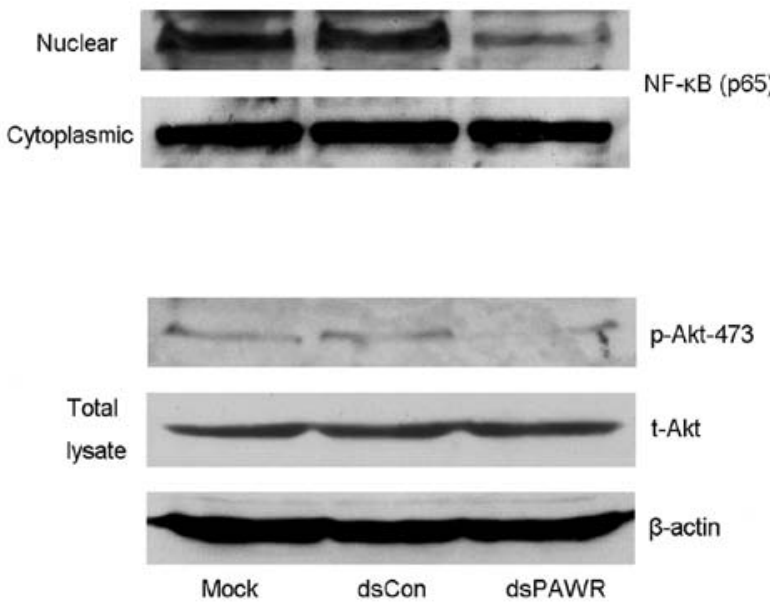

Figure 7. The molecular mechanism related to dsPAWR-433-induced cell apoptosis. DU145 cells were transfected with 50 nM dsRNA for 72 h. Representative blots from three independent experiments with identical results are shown. (A) The expression levels of pro-caspase- 9 and -8 were markedly decreased in the dsPAWR-433-treated DU145 cells. (B) Bcl-2 protein was reduced and Bax protein was not affected after dsPAWR-433 treatment. (C) The nuclear translocation of NF- $\mathrm{NB}$ and the phosphorylation of Akt were inhibited by dsPAWR-433 treatment.

level of pro-caspase- 3 was markedly decreased in the $50 \mathrm{nM}$ dsPAWR-433-treated DU145 cells at $72 \mathrm{~h}$ following treatment (Fig. 6C). Moreover, the 89 kDa cleaved PARP fragment was detected in the dsPAWR-433-treated samples. Thus, the significant changes in apoptosis-related proteins caused by dsPAWR-433 confirmed the ongoing apoptosis above and the 
anti-carcinogenic effects on the DU145 human prostate cancer cells.

The molecular mechanism related to dsPAWR-433-induced cell apoptosis. To examine which pathway plays a role in the dsPAWR-433-induced cell apoptosis, the cleavage of caspase- 8 and -9 was examined. As shown in Fig. 7A, the levels of pro-caspase- 8 and -9 were markedly decreased in the $50 \mathrm{nM}$ dsPAWR-433-treated DU145 cells at $72 \mathrm{~h}$ following treatment, indicating that both extrinsic and intrinsic pathways were active in the dsPAWR-433-treated cells.

PAWR-mediated apoptosis requires downregulation of Bcl-2 levels and PAWR regulates $\mathrm{Bcl}-2$ gene expression through a WT1-binding site in its promoter leading to a decrease in transcription $(27,28)$. Consistently, the expression of Bcl-2 was found to decrease in the dsPAWR-433-treated cells compared with the controls (Fig. 7B). However, the level of Bax, the pro-apoptotic member of the Bcl-2 family, was not altered after the treatment of dsPAWR-433.

Previous reseach has shown that PAWR is an important intersection in the network of tumor suppressors that involves the NF- $\mathrm{BB}$ and Akt pathways (29), which are both deregulated during prostate tumorigenesis. Thus, we detected these proteins and found that nuclear translocation of NF- $\kappa \mathrm{B}$ and phosphorylation of Akt were inhibited in the dsPAWR-433treated cells compared with the controls (Fig. 7C), which implied inactivation of these signaling pathways.

\section{Discussion}

RNA activation (RNAa) is an interesting and promising discovery of small RNA-mediated gene upregulation originally identified in several human cancer cell lines $(5,6)$. It may offer an alternative to manipulate gene expression potently and specifically if this phenomenon exists in most genes as RNAi and its rules could be deciphered. RNAa thus holds great promise as a therapeutic for reactivation of functionally silenced or low expressed TSGs in cancer patients. Our group and other investigators have obtained exciting results whereby upregulation of p21 by saRNA induced cell cycle arrest and apoptosis in human bladder cancer cells $(15,16)$ and renal cell carcinoma cells (17) in vitro, and inhibited the growth of xenograft prostate tumors (19) and orthotopic bladder tumors (20) in vivo.

Functional PAWR is a TSG essential for apoptosis and a cancer-selective target for cancer therapeutics (30), implicating its potential as a candidate for RNAa. Our attempts with dsRNAs targeting the PAWR promoter have successfully induced transcriptional activation of the PAWR gene in human cancer cells (14). Moreover, dsPAWR-435 actually induced growth inhibition of bladder and prostate cancer cells, suggesting that the increased PAWR protein by saRNA is physiologically functional and has great potential for the application in cancer therapy (14). In the present study, we demonstrated that another PAWR promoter targeted dsRNA, dsPAWR-433, could potently induce activation of PAWR gene expression in prostate cancer cells. Promisingly, MTT assay and flow cytometric analysis showed that dsPAWR-433 inhibited cell viability in a dose- and time-dependent manner and it was related to apoptotic cell death after treatment.
Apoptosis by ectopic PAWR involves activation of the Fas death receptor signaling pathway and concurrent nuclear factor- $\kappa \mathrm{B}(\mathrm{NF}-\kappa \mathrm{B})$ inhibition, which withdraws the anti-apoptotic roadblocks and allows the caspase cascade to proceed uninterrupted (23). PAWR induces apoptosis in hormone-independent cancer cells by enabling the translocation of Fas and Fas ligand (Fas/FasL) to the plasma membrane, which recruits the adapter protein Fas-dependent death domain (FADD), induces the formation of the death-inducing signaling complex (DISC), and thereby initiates the caspase- 8 dependent cascade (23). In parallel, PAWR translocates to the nucleus and inhibits NF- $\mathrm{B}$-mediated cell survival mechanism, which constitutes one of the mechanisms of PAWR-induced apoptosis (31). Moreover, PAWR has been shown to function in the cytoplasm, wherein it represses the tumor necrosis factor- $\alpha$-induced nuclear translocation of the p65 (Rel A) subunit by blocking the atypical protein kinase $\mathrm{C}(\mathrm{aPKC})$ or I $\mathrm{B}$ kinase $(\mathrm{IKK} \beta)$-mediated phosphorylation of the $\mathrm{NF}-\kappa \mathrm{B}$ inhibitory protein $\mathrm{I} \kappa \mathrm{B}$ (32). Activation of the Akt pathway is a frequent molecular event in human cancer and one of the major signaling pathways implicated in advanced prostate cancer $(33,34)$. Akt is also a direct substrate of aPKC, which places PAWR as a common step in the regulation of the Akt and NF- $\mathrm{B}$ pathways (35). These pathways regulate a number of pro-survival genes, including, but not limited to, anti-apoptotic genes such as those of the Bcl-2 family $(33,36)$. PAWR-mediated apoptosis requires downregulation of Bcl-2 levels and PAWR regulates Bcl-2 gene expression through a WT1-binding site in its promoter leading to a decrease in transcription $(27,28)$. Therefore, these interacting factors regulated by overexpressive PAWR promote the intracellular apoptotic cascade.

In the present study, two androgen-independent prostate cancer cell lines, DU145 and PC3, were chosen to test the antitumor effects of dsPAWR-433. Activation of PAWR gene expression by dsPAWR-433 not only activated the caspase8-dependent extracellular apoptotic pathway but also induced the caspase-9-dependent intracellular apoptosis by inhibition of Akt and NF- $\kappa$ B pathways and downregulation of Bcl-2 protein. Then, the activation of caspase- 3 plays a central role in apoptosis by cleaving intracellular proteins vital for cell survival and growth, such as PARP $(37,38)$, leading to the completion of apoptosis in the dsPAWR-433-treated prostate cancer cells.

To date, it appears difficult to discern the definite mechanisms of RNAa due to only a few genes activated and the diversity of the results from different genes. In contrast, we still have to screen multiple targets in order to activate a particular promoter. Regardless, RNAa offers a new approach to enhance endogenous gene expression and holds great promise as a therapeutic for reactivation of functionally silenced or lowly expressed TSGs in cancer patients. Despite the promise, further studies are needed to delineate the exact mechanism of RNAa and develop safe and effective in vivo saRNA delivery methods for clinical use.

\section{Acknowledgements}

The present study was supported by grants from the National Natural Science Foundation of China (grant no. 81101718) and 
the Natural Science Foundation of Zhejiang Province (grant nos. LY16H160012 and LY13H160009).

\section{References}

1. Siegel R, Ma J, Zou Z and Jemal A: Cancer statistics, 2014. CA Cancer J Clin 64: 9-29, 2014

2. Lares MR, Rossi JJ and Ouellet DL: RNAi and small interfering RNAs in human disease therapeutic applications. Trends Biotechnol 28: 570-579, 2010.

3. Castanotto D and Rossi JJ: The promises and pitfalls of RNA-interference-based therapeutics. Nature 457: 426-433, 2009.

4. Tiemann K and Rossi JJ: RNAi-based therapeutics-current status, challenges and prospects. EMBO Mol Med 1: 142-151, 2009.

5. Li LC, Okino ST, Zhao H, Pookot D, Place RF, Urakami S, Enokida H and Dahiya R: Small dsRNAs induce transcriptional activation in human cells. Proc Natl Acad Sci USA 103: 17337-17342, 2006

6. Janowski BA, Younger ST, Hardy DB, Ram R, Huffman KE and Corey DR: Activating gene expression in mammalian cells with promoter-targeted duplex RNAs. Nat Chem Biol 3: 166-173, 2007.

7. Place RF, Li LC, Pookot D, Noonan EJ and Dahiya R: MicroRNA-373 induces expression of genes with complementary promoter sequences. Proc Natl Acad Sci USA 105: 1608-1613, 2008.

8. Turunen MP, Lehtola T, Heinonen SE, Assefa GS, Korpisalo P, Girnary R, Glass CK, Väisänen S and Ylä-Herttuala S: Efficient regulation of VEGF expression by promoter-targeted lentiviral shRNAs based on epigenetic mechanism: A novel example of epigenetherapy. Circ Res 105: 604-609, 2009.

9. Huang V, Qin Y, Wang J, Wang X, Place RF, Lin G, Lue TF and Li LC: RNAa is conserved in mammalian cells. PLoS One 5: e8848, 2010.

10. Wang J, Place RF, Huang V, Wang X, Noonan EJ, Magyar CE, Huang $\mathbf{J}$ and Li LC: Prognostic value and function of KLF4 in prostate cancer: RNAa and vector-mediated overexpression identify KLF4 as an inhibitor of tumor cell growth and migration. Cancer Res 70: 10182-10191, 2010.

11. Matsui M, Sakurai F, Elbashir S, Foster DJ, Manoharan M and Corey DR: Activation of LDL receptor expression by small RNAs complementary to a noncoding transcript that overlaps the LDLR promoter. Chem Biol 17: 1344-1355, 2010.

12. Wang X, Wang J, Huang V, Place RF and Li LC: Induction of NANOG expression by targeting promoter sequence with small activating RNA antagonizes retinoic acid-induced differentiation. Biochem J 443: 821-828, 2012.

13. Wang T, Li M, Yuan H, Zhan Y, Xu H, Wang S, Yang W, Liu J, Ye $Z$ and Li LC: saRNA guided iNOS up-regulation improves erectile function of diabetic rats. J Urol 190: 790-798, 2013.

14. Yang K, Shen J, Xie YQ, Lin YW, Qin J, Mao QQ, Zheng XY and Xie LP: Promoter-targeted double-stranded small RNAs activate PAWR gene expression in human cancer cells. Int J Biochem Cell Biol 45: 1338-1346, 2013

15. Chen Z, Place RF, Jia ZJ, Pookot D, Dahiya R and Li LC: Antitumor effect of dsRNA-induced $\mathrm{p} 21^{\mathrm{WAF} / \mathrm{ClP} 1}$ gene activation in human bladder cancer cells. Mol Cancer Ther 7: 698-703, 2008.

16. Yang K, Zheng XY, Qin J, Wang YB, Bai Y, Mao QQ, Wan Q, Wu ZM and Xie LP: Up-regulation of p21 $1^{\text {WAF1/Cipl by saRNA }}$ induces G1-phase arrest and apoptosis in T24 human bladder cancer cells. Cancer Lett 265: 206-214, 2008.

17. Whitson JM, Noonan EJ, Pookot D, Place RF and Dahiya R Double stranded-RNA-mediated activation of P21 gene induced apoptosis and cell cycle arrest in renal cell carcinoma. Int J Cancer 125: 446-452, 2009.

18. Wei J, Zhao J, Long M, Han Y, Wang X, Lin F, Ren J, He T and Zhang H: p21WAF1/CIP1 gene transcriptional activation exerts cell growth inhibition and enhances chemosensitivity to cisplatin in lung carcinoma cell. BMC Cancer 10: 632, 2010.
19. Place RF, Wang J, Noonan EJ, Meyers R, Manoharan M, Charisse K, Duncan R, Huang V, Wang X and Li LC: Formulation of small activating RNA into lipidoid nanoparticles inhibits xenograft prostate tumor growth by inducing p21 expression. Mol Ther Nucleic Acids 1: e15, 2012.

20. Kang MR, Yang G, Place RF, Charisse K, Epstein-Barash H, Manoharan M and Li LC: Intravesical delivery of small activating RNA formulated into lipid nanoparticles inhibits orthotopic bladder tumor growth. Cancer Res 72: 5069-5079, 2012.

21. Johnstone RW, Tommerup N, Hansen C, Vissing H and Shi Y: Mapping of the human PAWR (par-4) gene to chromosome 12q21. Genomics 53: 241-243, 1998.

22. Sells SF, Wood DP Jr, Joshi-Barve SS, Muthukumar S, Jacob RJ, Crist SA, Humphreys S and Rangnekar VM: Commonality of the gene programs induced by effectors of apoptosis in androgendependent and -independent prostate cells. Cell Growth Differ 5: 457-466, 1994.

23. Chakraborty M, Qiu SG, Vasudevan KM and Rangnekar VM: Par-4 drives trafficking and activation of Fas and Fasl to induce prostate cancer cell apoptosis and tumor regression. Cancer Res 61: 7255-7263, 2001.

24. Gurumurthy S, Goswami A, Vasudevan KM and Rangnekar VM: Phosphorylation of Par- 4 by protein kinase A is critical for apoptosis. Mol Cell Biol 25: 1146-1161, 2005

25. Goswami A, Burikhanov R, de Thonel A, Fujita N, Goswami M, Zhao Y, Eriksson JE, Tsuruo T and Rangnekar VM: Binding and phosphorylation of par-4 by akt is essential for cancer cell survival. Mol Cell 20: 33-44, 2005.

26. El-Guendy N, Zhao Y, Gurumurthy S, Burikhanov R and Rangnekar VM: Identification of a unique core domain of par-4 sufficient for selective apoptosis induction in cancer cells. Mol Cell Biol 23: 5516-5525, 2003.

27. Qiu G, Ahmed M, Sells SF, Mohiuddin M, Weinstein MH and Rangnekar VM: Mutually exclusive expression patterns of Bcl-2 and Par-4 in human prostate tumors consistent with downregulation of Bcl-2 by Par-4. Oncogene 18: 623-631, 1999.

28. Cheema SK, Mishra SK, Rangnekar VM, Tari AM, Kumar R and Lopez-Berestein G: Par-4 transcriptionally regulates Bcl-2 through a WT1-binding site on the $b c l-2$ promoter. J Biol Chem 278: 19995-20005, 2003.

29. Diaz-Meco MT and Abu-Baker S: The Par-4/PTEN connection in tumor suppression. Cell Cycle 8: 2518-2522, 2009.

30. Goswami A, Ranganathan P and Rangnekar VM: The phosphoinositide 3-kinase/Akt1/Par-4 axis: A cancer-selective therapeutic target. Cancer Res 66: 2889-2892, 2006.

31. Nalca A, Qiu SG, El-Guendy N, Krishnan S and Rangnekar VM: Oncogenic Ras sensitizes cells to apoptosis by Par-4. J Biol Chem 274: 29976-29983, 1999.

32. Diaz-Meco MT, Lallena MJ, Monjas A, Frutos S and Moscat J: Inactivation of the inhibitory kappaB protein kinase/nuclear factor kappaB pathway by Par-4 expression potentiates tumor necrosis factor alpha-induced apoptosis. J Biol Chem 274: 19606-19612, 1999.

33. Manning BD and Cantley LC: AKT/PKB signaling: Navigating downstream. Cell 129: 1261-1274, 2007.

34. Malik SN, Brattain M, Ghosh PM, Troyer DA, Prihoda T, Bedolla R and Kreisberg JI: Immunohistochemical demonstration of phospho-Akt in high Gleason grade prostate cancer. Clin Cancer Res 8: 1168-1171, 2002.

35. Joshi J, Fernandez-Marcos PJ, Galvez A, Amanchy R, Linares JF, Duran A, Pathrose P, Leitges M, Cañamero M, Collado M, et al: Par-4 inhibits Akt and suppresses Ras-induced lung tumorigenesis. EMBO J 27: 2181-2193, 2008.

36. Barkett $M$ and Gilmore TD: Control of apoptosis by Rel/NF-kappaB transcription factors. Oncogene 18: 6910-6924, 1999.

37. Salvesen GS and Dixit VM: Caspase activation: The inducedproximity model. Proc Natl Acad Sci USA 96: 10964-10967, 1999.

38. Ivana Scovassi A and Diederich M: Modulation of poly(ADP-ribosylation) in apoptotic cells. Biochem Pharmacol 68: 1041-1047, 2004. 